\title{
Wollen und nicht können
}

\section{Malnutritionsmanagement in medizinischen Kliniken eines schweizerischen Zentrumsspitals: Erleben und Sichtweisen von Patient(inn)en}

Elisabeth Haldemann-Jenni ${ }^{1,2}$ (MScN, RN), Katharina Fierz ${ }^{2}(P h D, R N)$, Irena Anna Frei ${ }^{2,3}(P h D, R N)$

${ }^{1}$ Fachbereich Gesundheit, Hochschule für Angewandte Wissenschaften, FHS St. Gallen

${ }^{2}$ Departement Public Health, Pflegewissenschaft, Universität Basel

${ }^{3}$ Abteilung Praxisentwicklung, Universitätsspital Basel

\begin{abstract}
Zusammenfassung:
Hintergrund: Malnutrition ist ein Phänomen, das bei Patient(inn)en in Gesundheitsinstitutionen häufig vorkommt und für die Betroffenen mit negativen gesundheitlichen Konsequenzen verbunden ist. In vielen Spitälern wurden deshalb Malnutritionsprogramme entwickelt und etabliert, jedoch in der Regel ohne die Perspektive der Betroffenen miteinzubeziehen. Ob ein Programm den tatsächlichen Bedürfnissen der Betroffenen entspricht, kann bis heute lediglich aus Alltagsgesprächen abgeleitet werden.

Ziel: Deshalb war das Ziel der Studie, die Bedürfnisse von Betroffenen in Bezug auf ihr Malnutritionsmanagement und ihr Erleben der angebotenen Ernährungsinterventionen zu explorieren.

Methode: Es wurde ein qualitativer, induktiver Ansatz gewählt. Von September 2011 bis Mai 2012 wurden mit acht Patient(inn) en leitfadengestützte Interviews geführt und nach Mayring inhaltsanalytisch ausgewertet.

Resultate: Die Patient(inn)en bewegten sich bei der Nahrungsaufnahme im Spannungsfeld zwischen «Wollen» und «Nicht können». Unter anderem erschwerten Schmerzen beim Kauen und Schlucken, Übelkeit oder verändertes Geschmacksempfinden die Nahrungsaufnahme. Daneben trafen Betroffene auf institutionelle Hindernisse, wie vorgegebene Essenszeiten und eine eingeschränkte Menüauswahl. Die Patient(inn)en bewältigten ihre Ernährungsprobleme mehrheitlich selber.

Schlussfolgerungen: Pflegefachpersonen sollten befähigt werden, Patient(inn)en in ihrem Malnutritionsmanagement effektiver zu unterstützen. Zugleich sind institutionelle Hindernisse zu eliminieren, damit sich Patient(inn)en individueller ernähren können.
\end{abstract}

Schlüsselwörter: Malnutrition, Patientenerfahrung, Selbstmanagement, Qualitative Forschung

\section{Between intent and inability - Management of malnutrition on medical wards of a centre hospital in Switzerland: patients' experiences and perceptions}

\section{Abstract:}

Background: Malnutrition is a common phenomenon in acute care institutions accounting for many negative health consequences for the patient. In many hospitals, therefore, malnutrition risk screening and nutrition management programs were established; however, programs were commonly developed without integrating the patients' perspective. It is unknown if the program covers the patients' needs and if the interventions are worthwhile.

Aim: Because patient experience is known solely from everyday conversations, the aim of the study was to explore affected patients' experience regarding nutrition management.

Method: The study has a qualitative, inductive approach. From September 2011 till May 2012, seven women and one man were interviewed. To analyse the guided interviews, content analysis was used.

Results: The analysis shows that patients find themselves between 'to want but not be able to eat'. Patients at risk encounter barriers due to their physical condition, such as swallowing- and chewing pain, nausea and dysgeusia and barriers associated with the system when ordering meals, such as fixed mealtimes, a limited variety of the menu and non-tasting supplements. To overcome these barriers patients are left to develop self-management strategies and to be in charge of their nutrition. Conclusions: Therefore, targeted training for caregivers is a key, enabling them to support patients individually in their nutrition management. At the same time, institutional barriers must be removed.

Keywords: malnutrition, patients experience, self-management, qualitative research 
Was ist (zu dieser Thematik) schon bekannt?

In vielen Institutionen wurden Malnutritionsprogramme etabliert, um Malnutrition bei schwer kranken Menschen zu verhindern bzw. zu lindern.

Was ist neu?

Trotz standardisierter Programme müssen Patient(inn)en ihr Malnutritionsmanagement selber steuern.

Welche Konsequenzen haben die Ergebnisse für die Pflegepraxis? Die Pflegenden sollten sich ihrer Rolle im Malnutritionsprogramm bewusst sein. Gezielt geschulte Pflegepersonen könnten die Situation der Patient(inn)en verbessern.

\section{Einleitung}

Jeder fünfte Patient in Schweizer Akutspitälern ist von einem Malnutritionsrisiko oder von Malnutrition betroffen. In einer an medizinischen Kliniken von sieben mittelgroßen Spitälern in der Schweiz durchgeführten Studie ist belegt, dass 18,2\% von 32837 mittels Nutritional Risk Screening 2002 (NRS-2002) erfassten Patient(inn)en malnutriert waren oder ein hohes Risiko für Malnutrition aufwiesen (Imoberdorf et al., 2010). Auch internationale Studien beschreiben Raten von 20 bis 40 \% für ein Malnutritionsrisiko (Kondrup, Allison, Elia, Vellas \& Plauth, 2003a; Kyle et al., 2003; Ockenga et al., 2005). Häufig verschlechtert sich der Ernährungszustand bei Patient(inn)en während eines Spitalaufenthalts (Kondrup et al., 2002). Die physiologischen Auswirkungen einer Malnutrition sind für die Betroffenen einschneidend. Die Patienten(inn)en mit nachgewiesener Malnutrition weisen eine höhere Mortalität auf und haben häufiger Komplikationen wie Infekte oder längere Wundheilungsphasen (Correia \& Waitzberg, 2003; Van Venrooij, De Vos, Borgmeijer-Hoelen, Haaring \& De Mol, 2008). Dadurch verlängert sich die Hospitalisationsdauer (Kondrup et al., 2002; Kyle, Pirlich, Schuetz, Lochs \& Pichard, 2004; Sorensen et al., 2008) und die Spitalkosten steigen (Correia \& Waitzberg, 2003; Ockenga et al., 2005). In der Schweiz beliefen sich 2004 die auf Malnutrition zurückzuführenden Kosten auf über 526 Millionen Franken (Frei, 2006). Risikopatienten, die Ergänzungsnahrung einnahmen, zeigten bis zu viermal weniger Komplikationen (Stratton \& Elia, 2007) und waren kürzer hospitalisiert als die jeweilige Kontrollgruppe (Johansen et al., 2004; Stratton \& Elia, 2007). Im Wissen, dass sich der Ernährungszustand durch geeignete Interventionen zur Nahrungsunterstützung verbessern lässt, sind eine systematische Risikoerfassung und ein gezieltes Management der Ernährung von entscheidender Bedeutsamkeit, um Malnutrition zu verhindern bzw. zu lindern (Beck et al., 2001; Kondrup et al., 2003b; Sorensen et al., 2008).

\section{Das Malnutritionsprogramm}

Das Malnutritionsprogramm der medizinischen Kliniken in einem Schweizer Zentrumsspital besteht aus einer pfle- gegeleiteten, systematischen Risikoeinschätzung und, falls notwendig, dem darauf folgenden Malnutritionsmanagement, welches ein Set von spezifischen Interventionen umfasst.

Die Risikoeinschätzung wird mittels des Nutrition Risk Screenings 2002 (NRS-2002) bei allen ein- oder übertretenden Patient(inn)en durchgeführt. Die European Society for Clinical Nutrtition and Metabolism (ESPEN) empfiehlt das NRS-2002, um bei hospitalisierten Patient(inn)en ein leichtes bis schweres Risiko für Malnutrition zu erfassen (Kondrup et al., 2003a). Mit diesem Fragebogen ist es möglich, einen ungewollten Gewichtsverlust, das Essverhalten vor Spitaleintritt, den BodyMass-Index (BMI), das Alter ( $\geq 70$ ) und den Krankheitsmetabolismus zu erfassen. Je nach Gewichtung wird ein Score von 0-7 Punkten errechnet, wobei 0 kein Risiko und 7 das höchstmöglichste Risiko bedeutet (Kondrup et al., 2003b). Lässt sich ein Risiko feststellen, erfolgt das Malnutritionsmanagement. Dieses Set von Interventionen entstand in interprofessioneller und -disziplinärer Zusammenarbeit von Ernährungsberaterinnen, Pflegefachpersonen, Internisten und Endokrinologen. Es beinhaltet das Führen eines Ess- und Trinkprotokolls, das Anbieten von Zwischenmahlzeiten, die Abgabe einer Informationsbroschüre und bei schwieriger Ernährungssituation bzw. bei Patient(inn)en mit einem Score $\geq 3$ das Hinzuziehen der Ernährungsberatung und des Arztes/ der Ärztin. Bei ärztlich bestätigter Malnutrition oder einem Malnutritionsrisiko bietet die Küche zusätzliche Menükomponenten an, beispielsweise angereicherte Suppen. Die Pflegenden besuchen jedes Jahr einen Refresherkurs, damit sie bezüglich der Malnutrition und des Malnutritionsprogramms auf dem neusten Stand sind.

Erfahrungen von Patient(inn)en, welche ein Screening und Interventionen bezüglich Malnutrition erhielten, sind in der nationalen und internationalen Forschungsliteratur kaum beschrieben. Ferguson, Capra, Bauer und Banks (2001) erfassten die Patientenzufriedenheit mit einem selbst entwickelten Instrument und zeigten einen signifikant positiven Zusammenhang zwischen der Patientenzufriedenheit, den Interventionen des Ernährungsservices und der Betreuung respektive Kompetenz der Ernährungsberatung auf.

Um das Malnutritionsprogramm optimieren zu können, ist es für das interprofessionelle und interdisziplinäre Behandlungsteam von zentraler Bedeutung, wie Patient(inn)en mit einem festgestellten Malnutritionsrisiko ihre Ernährung und das dazu gehörende Malnutritionsmanagement erleben. Dies ist jedoch ausschließlich von der Sicht der Betroffenen abhängig. Darum war das Ziel der Studie, das Erleben von Patient(inn)en mit einem Malnutritionsrisiko bezüglich des Malnutritionsprogramms im Spital zu erfassen. Folgende Forschungsfrage stand im Zentrum: Wie erleben erwachsene Patient(inn)en mit einem Malnutritionsrisiko ihre Ernährung und das dazu gehörende Management während ihres stationären Aufenthalts? 


\section{Methode}

\section{Design}

Um diese Studie durchzuführen, wählten wir den deskriptiven, qualitativen Ansatz. Dadurch lässt sich induktiv explorieren, welche Erfahrungen Patient(inn)en mit dem Malnutritionsprogramm machen, denn sie sind die «wirklich Wissenden und Kennenden» ihrer Wahrnehmung (McIntyre, 2008; 11). Wir befragten Patient(inn)en, die im Rahmen eines routinemäßig durchgeführten Screenings ein Malnutritionsrisiko zeigten und anschließend Interventionen gemäß Malnutritionsmanagement erhielten. Sie mussten über 18 Jahre sein, über mündliche und schriftliche Deutschkenntnisse verfügen und in der Lage sein, an einem Gespräch von mindestens einer halben Stunde aktiv teilzunehmen. Nicht berücksichtigt wurden Patient(inn)en, die an einer ICD-10 diagnostizierten Essstörung litten.

Die Pflegeexpertinnen der jeweiligen Abteilungen fragten gemäß den Einschlusskriterien geeignete Patient(inn)en an und übergaben ihnen die Informationsunterlagen. Nach 24 Stunden fragte die betreuende Pflegefachperson nochmals nach und bei definitivem Einverständnis benachrichtigte sie das Studienteam. Die interessierten Patient(inn)en erhielten zu Beginn des Interviews noch einmal Informationen bezüglich der Studie und unterschrieben eine schriftliche Einwilligungserklärung.

\section{Datensammlung und Analyse}

Für die Datensammlung befragten wir alle Teilnehmer(inn)en während des Spitalaufenthaltes anhand eines halbstrukturierten Leitfadens in einem geschützten Raum. Wir formulierten offene Fragen zu den Themen «Erfahrungen im Allgemeinen mit den Mahlzeiten im Spital»; «Informationen in Bezug auf das Malnutritionsrisiko»; «Erleben der Interventionen des Malnutritionsmanagements» und «Erfahrungen mit den Pflegenden im Umgang mit den Interventionen».

Die Interviews zeichneten wir auf einen elektronischen Datenträger auf, transkribierten sie in die Schriftsprache und werteten sie mithilfe der qualitativen Inhaltsanalyse nach Mayring (2010) aus. Datensammlung und Analyse verliefen parallel, damit sich der Interviewleitfaden mit neuen Erkenntnissen aus den Interviews ergänzen ließ. Es ergaben sich bei den weiteren Interviews durchaus neue, jedoch auch redundante Aussagen.

Das Vorgehen nach Mayring erlaubte es, die transkribierten Interviews induktiv, systematisch und regelgeleitet inhaltsanalytisch so zu reduzieren, dass die wesentlichen Inhalte erhalten blieben. Das geschah durch Paraphrasieren, Reduzieren und Zusammenfassen der Textteile. Aus den zusammengefassten Textteilen bildeten wir Kategorien, welche wir definiert mit Codes und
Ankerbeispielen hinterlegten. Nach Bearbeitung der ersten vier Interviews analysierten wir mithilfe der Kategorien die restlichen Interviews. Tauchten darin neue Aspekte auf, prüften wir die bereits analysierten Interviews nochmals auf diese Inhalte hin. Während der Analyse dienten Fachliteratur oder Lexika zur Explikation einzelner interpretationsbedürftiger Textstellen. Um eine verfälschte Wiedergabe zu verhindern, verglichen wir die Ergebnisse mit den Originalinterviews. Die soziodemografischen und medizinischen Daten der Teilnehmer(inn)en erfassten wir deskriptiv.

\section{Datenschutz und Qualitätssicherung}

Alle erhobenen Daten anonymisierten wir. Zur Qualitätssicherung legten wir die Daten in den verschiedenen Analyseschritten einer Reflexionsgruppe und den Studienbegleiterinnen zur kritischen Diskussion vor. Die Studie wurde von der zuständigen Ethikkommission begutachtet und als unbedenklich erklärt.

\section{Ergebnisse}

\section{Beschreibung der Studienteilnehmenden}

Im Zeitraum zwischen September 2011 und Ende Mai 2012 erfolgte die Anfrage von insgesamt 36 Patient(inn)en. 26 sagten direkt ab, zwei nach der weiteren Anfrage nach 24 Stunden. Letztlich konnten wir sieben Frauen und einen Mann befragen. Das Alter der Teilnehmenden lag zwischen 34 und 93 Jahren. Vier Teilnehmer(inn)en wohnten alleine, vier lebten mit Partnern respektive Angehörigen. Der Score des Screenings mit NRS-2002 lag zwischen zwei und vier, der Body-Mass-Index zwischen 15 und 30. Fünf Patient(inn)en litten unter Nebenwirkungen onkologischer Therapien, eine Patientin litt an den Folgen einer Pneumonie und zwei Patient(inn)en waren wegen ihres verschlechterten Allgemeinzustands hospitalisiert. Alle nahmen zwischen fünf und 25 Medikamente ein oder erhielten mehrere Medikamente parenteral. Bei allen Patient(inn)en erfolgte eine Einschätzung durch NRS-2002 und sie erhielten Interventionen gemäß Malnutritionsmanagement. Eine Patientin hatte die Informationsbroschüre erhalten, welche im Malnutritionsmanagement bei festgestelltem Malnutritionsrisiko erwähnt ist. Zwei Patient(inn)en sagten, dass sie diese Broschüre nicht erhalten haben. Die verbleibenden fünf Patient(inn)en konnten sich nicht erinnern. Bei zwei Patient(inn)en war zum Zeitpunkt des Interviews die Ernährungsberatung involviert. Eine Patientin war gemäß Eintrag im Screeningdokument für die Ernährungsberatung angemeldet. Als Zwischenmahlzeit erhielten alle Patient(inn)en ausschließlich Trinknahrungen. 


\section{Ergebnisse aus den Interviews}

Themen, die das Erleben des Malnutritionsprogramms bestimmen, sind auf der persönlichen Ebene «Leiden am und beim Essen» sowie «Bewältigungsstrategien» und auf der betrieblichen Ebene «Erfragen des Ernährungszustands»; «Beratung durch die Pflegeperson»; «Auswahl der Mahlzeiten» und «Interventionen analog des Malnutritionsmanagements».

\section{Persönliche Ebene}

Die Studienteilnehmer(inn)en bewegten sich in Bezug auf ihre Ernährung im Spannungsfeld zwischen «Wollen» und «Nicht können». Alle Patient(inn)en erklärten, dass ihnen Essen viel bedeute, da es etwas Schönes und für den Sozialkontakt etwas Wichtiges sei. Sie erwähnten, dass sie eigentlich gerne essen würden, doch dies sei mit sehr viel Mühe verbunden.

\section{Leiden am und beim Essen}

Fünf der befragten Patient(inn)en litten infolge ihrer Erkrankung an veränderten Geschmacksempfindungen. Sie beklagten, dass das Essen metallisch, chemisch und/oder bitter schmecke. Sie klagten, trotz Medikamenten an Emesis und Nausea zu leiden, und Ekel dem Essen gegenüber zu empfinden. Wegen einer bestehenden Mukositis hatten sie starke Schluckschmerzen. Eine Patientin erklärte, dass sie wegen starker Dyspnoe kaum Nahrung zu sich nehmen könne. Die Befragten erwähnten auch, dass das Essen für sie anstrengend sei. Dies erschwere die Nahrungsaufnahme oder mache sie sogar unmöglich. Eine Patientin, welche schließlich parenteral ernährt werden musste, beschrieb die Zeit davor folgendermaßen: «Essen ist für mich immer etwas Positives und etwas Schönes gewesen. Während der Chemozeit habe ich eine extreme Dysgeusie entwickelt. Alles war metallisch, alles war salzig, alles war bitter. Viele Lebensmittel gingen nicht mehr. Ich habe schon dort sehr viel Lebensqualität eingebüßt. Letztlich hat es mir den Bogen gegeben, da wirklich alles eklig war und mir dazu noch ständig übel ist.» (I: 8, 154-160)

Alle Patient(inn)en erwähnten das Phänomen «plötzlich genug» und «Es kommt wieder hoch». Diese Empfindungen entstanden durch starke Schmerzen und/oder durch Essen, das nicht mehr so schmeckte, wie sie es gewohnt waren. Einige haben versuchten, über ihre innere Barriere hinweg zu essen und mussten daraufhin erbrechen. «Ich weiß nicht, es macht mir da (sie zeigt auf die Höhe des Sternums) wie ein Türchen zu. Und wenn ich mich dann zwinge, kommt alles wieder retour.» (I: 3, 30-32) Die meisten Teilnehmer(inn)en beschrieben, dass sie das präsentierte Essen zwar gelüstet und sie Verlangen nach Süßem oder Salzigem nach wie vor wahrnehmen. Durch die gustatorischen Veränderungen und die funktionellen Erschwernisse vergehe ihnen jedoch beim Essen die Lust. Nur eine einzige Teilnehmerin sagte, dass keine Gelüste nach irgendetwas vorhanden seien. Alle Patient(inn)en wussten, dass sie essen sollten, um bei Kräften zu bleiben. Eine Patientin sagte: «Ich hätte gerne etwas, das ich nehmen könnte, damit ich wieder zu Kräften komme.» (I: 1, 366) Eine andere Patientin meinte, dass sie auf der Menükarte gerne Informationen zur Nahrungszusammensetzung gelesen hätte. Sie wisse zwar, was eine ausgewogene Ernährung ausmache, aber es sei ihr nicht bekannt, wie viel Fett, Eiweiß und Kohlenhydrate in den einzelnen Menükomponenten enthalten seien. Die Patient(inn)en machten sich Sorgen, wie sie ihren Nahrungsbedarf decken können, wenn sie so wenige Lebensmittel zu sich nehmen und so einseitig essen.

\section{Bewältigungsstrategie}

Die Teilnehmenden versuchten, sich mit ihren funktionalen und gustatorischen Einschränkungen zu arrangieren. Sie probierten verschiedene Möglichkeiten und Nahrungsmittel aus, damit sie trotz Einschränkungen Nahrung zu sich nehmen konnten. Mehrere Patient(inn)en sagten, dass ihnen zwar breiig zubereitete Nahrung widerstrebe, sie wussten jedoch, dass sie im Moment nur solche schlucken konnten. Sie wählten vor allem Essen, das «runter rutscht»: «Ich weiß genau, wie viel ich essen kann. Ich kriege eine Suppe, das ist das Beste, die rutscht immer.» (I: 5, 59) Nicht immer konnten sich die Teilnehmenden darauf verlassen, dass das Essen, welches sie bestellten, ausreichend schluckfähig war. Damit sie trotzdem essen konnten, behalfen sie sich mit Tricks, beispielsweise einen Schluck Wasser oder Suppe zu nehmen, um das Essen schluckfähiger zu machen: «Ich habe immer ein Glas Wasser dabei, oder wenn ich noch Suppe übrig habe, dann nehme ich noch einen Löffel, damit es runter rutscht.» (I: 3, 58-60) Manche setzten sich an den Bettrand oder an den Tisch, um durch die aufrechte Position den Schluckvorgang zu erleichtern. Eine andere Patientin erzählte, dass sie das Essen kalt werden ließ, denn kühle oder kalte Nahrungsmittel schienen besser schluckfähig zu sein: "Ich habe keinen Hunger, (aber) so einen Kompott kann man gut essen. Er ist auch saftig.» (I: 7, 110-112) Manchmal erhielten sie vor dem Essen Schmerzmedikamente, die aber nicht immer genügend halfen. Zum Umgang mit der gustatorischen Fehlempfindung meinte eine Patientin: «Also ich esse einfach, damit ich gegessen habe. Das muss ich schon sagen.» (I: 6, 227-229)

Während der Interviews kam die Frage auf, was den Befragten dabei helfen könnte, ihre Ernährungssituation zu verbessern. Einige Patient(inn)en konnten nichts Bestimmtes nennen, andere hatten konkrete Wünsche, beispielsweise mehrere kleinere Mahlzeiten pro Tag. Sie meinten, dass sie dadurch mehr Nahrung zu sich nehmen könnten als mit drei großen. Es war ihnen nie möglich, alles zu essen, bevor ihr Körper ihnen wieder einen Strich durch die Rechnung machte. Sie wünschten sich flexiblere Essenszeiten, um Nahrung zu sich nehmen zu können, wenn sie Hunger und/oder Lust verspürten: «Ich habe mir manchmal gewünscht, dass ich vielleicht, sagen wir, um zwölf ein paar Bissen hätte essen können und vielleicht um ein Uhr eine Kleinigkeit hätte essen können.» (I: 1, 969-700) 


\section{Betriebliche Ebene}

\section{Das Erfragen des Ernährungszustands}

Das pflegegeleitete Malnutritionsprogramm beginnt schon mit der Eintrittsanamnese. Dabei erfragt die Pflegefachperson die Ernährungssituation vor dem Spitaleintritt. Die meisten Patient(inn)en konnten sich jedoch nicht mehr an diese speziellen Fragen erinnern: «Daran kann ich mich nicht mehr erinnern. (...) Es wird so viel gefragt.» (I: 1, 274-294) Patient(inn)en, die sich noch erinnern konnten, sagten, die Befragung sei adäquat und sachlich gewesen. Sie erklärten aber, dass die Informationen über ihr Malnutritionsrisiko bei ihnen keine Reaktion ausgelöst habe, weil sie sich nicht vorstellen konnten, was das für sie bedeutet: «Die Bedeutung der Mangelernährung realisiert man erst, wenn sie da ist. Das ist nichts, das man bei einem Vorbereitungsgespräch oder beim Eintrittsgespräch (...) klar machen kann.» (I: 8, 626-632)

\section{Beratung durch die Pflegepersonen}

Die Patient(inn)en sprachen nicht von Beratung, wenn die Pflegeperson die Bestellung für das Essen aufnahm. «Es sind Pflegeassistentinnen, die kommen. Über die Ernährung sagen sie nichts. Die lassen einen machen. Sie sagen einfach 〈Worauf haben Sie Lust? Was möchten Sie gerne?> Aber Beratung ist keine da.» (I: 6, 312-317) Die Pflegeperson las ihnen die Menüauswahl vor oder schlug einzelne Komponenten vor. Die meisten Patient(inn)en bevorzugten es jedoch, die Menüvorschläge selbst lesen zu können. Sie nahmen wahr, dass es den Pflegepersonen wichtig war, ob die Mahlzeit geschmeckt hatte oder nicht und auch, welche Menge sie gegessen hatten. Die Wichtigkeit der Mahlzeiten stand aber ihrer Meinung nach nicht im Vordergrund, weil die Pflegepersonen anderes zu tun hätten, was prioritärer sei. Eine Patientin formulierte es so: «Ich glaube, solange der Patient isst und ernährt ist, ist es der Pflege häufig, ich sage jetzt dem, nicht gerade 〈egal〉. Aber es ist ihnen recht, wenn es einfach ein Selbstläufer ist. Wenn das über die Pflegeassistentinnen läuft (...) und es erledigt ist.» (I: 8, 409-413)

Die Pflegepersonen haben die Patient(inn)en nicht auf die einzelnen speziellen Menükomponenten für Malnutritionspatient(inn)en aufmerksam gemacht, obwohl diese aus den Schulungen zum Malnutritionsprogramm bekannt sein sollten.

\section{Auswahl der Mahlzeiten}

Weil die Patient(inn)en vor allem leicht schluckbare Speisen benötigten, war die tägliche Auswahl aus der Menükarte beschränkt: «Ja, es gibt schon Schwierigkeiten, wenn man nicht mehr weiß, was.» (I: 3, 444) Die Patient(inn)en wählten vor allem Suppen, Kartoffelpüree, Grießbrei, Saucen und Kompotts. Zwei Teilnehmende bedauerten, dass sie nicht alles jederzeit wählen konnten. Eine Privatpatientin konnte ihre Mahlzeiten aus der gesamten Menükarte zusammensetzen. Das war bei den allgemeinversicherten Patient(inn)en nicht möglich. Bei zwei Patient(inn)en beobachteten wir während des Interviews, dass die bestell- ten Kompotte nicht geliefert wurden: «Oh, und wo ist mein Apfelmus? Den haben sie vergessen. Oh nein, das glaube ich jetzt nicht. Den hätte ich schon gerne.» (I: 6, 731-733) Auf Nachfragen hin erhielten sie die Antwort, dass keine Bestellung erfolgt sei. $\mathrm{Zu}$ beobachten war auch, dass zwei Patient(inn)en nicht das bestellte Menü erhielten.

Interventionen analog des Malnutritionsmanagements Im Malnutritionsmanagement ist vorgesehen, ein Ess- und Trinkprotokoll zu führen. Bis auf zwei Patient(inn)en wussten alle, dass ein Protokoll geschrieben wurde, respektive drei Patient(inn)en füllten es selbstständig aus: «Ja, sie haben da so ein Blatt gehabt. Und dann haben sie geschaut, wie viel ich gegessen habe, und dann haben sie es aufgeschrieben.» (I: 4, 502-503) Alle Patient(inn)en bestätigen, das Aufschreiben der Ess- und Trinkmenge sei gut, weil die Pflegepersonen Kontrolle darüber haben müssten. Sie erzählten, dass die Pflegepersonen sie nicht fragten, sondern die Kontrolle lediglich durch das Heben des Wärmedeckels geschehe. Dies störte sie aber nicht. Die Befragten sagten jedoch auch, dass sie das nicht animiere, mehr zu essen. Eine Patientin wies auf den Widerspruch hin, dass die Menge zwar aufgeschrieben werde, ohne jedoch die Portionsgröße festzuhalten.

Das Malnutritionsmanagement legt die Abgabe von Trinknahrung an die betroffenen Patienten fest. Die Teilnehmenden beurteilen die Trinknahrungen nach $\mathrm{Ge}$ schmack und Konsistenz. So meinte eine Patientin: "Ich denke, dass die eine Möglichkeit sind, weil diese rutschen. Man hat mit wenig Volumen eine hohe Kalorienzahl. Das Problem ist einfach, dass ich sie nicht trinkbar finde (...), sie sind zu konzentriert, zu chemisch.» (I: 2, 461/695-698) Oft erhielten die Patient(inn)en die Trinknahrung im Laufe des Vormittags oder Nachmittags mit der Aufforderung, diese zu trinken. Die meisten Patient(inn)en empfanden Trinknahrung jedoch als so sättigend, dass dadurch der Appetit und die Einnahme der regulären Mahlzeit stark beeinträchtigt waren.

Mehrmals erwähnten die Patient(inn)en, der Geschmack und die Temperatur der Trinknahrung könne die Einnahme beeinflussen. Auf die Frage, wie diese zu servieren seien, waren alle der Meinung, dass sie kühl oder kalt sein sollten. Eine Patientin erklärte, dass sie Eis oder kaltes Wasser verlange, um die Getränke zu kühlen oder zu verdünnen. Nur eine Patientin erhielt die Trinknahrung im Glas, allen anderen Befragten in der Originalverpackung. Eine Patientin meinte dazu: «Die Pflegenden könnten kreativer sein.» (I: 2, 647) Einige Patient(inn)en hatten auch die Idee, zu Beginn anhand von kleinen Portionen den Lieblingsgeschmack eruieren zu können.

Die Frage, ob sie auch andere Zwischenmahlzeiten als Trinknahrungen angeboten erhielten, verneinten alle Patient(inn)en.

\section{Diskussion}

Für alle Teilnehmenden ist Essen an und für sich etwas Schönes. Die Verminderung von Genuss und Freude am 
Essen führten bei der Nahrungsaufnahme zu einem Problem, welches einer Lösung bedurfte. Die Patient(inn)en befanden sich bei jeder Mahlzeit zwischen «Wollen» und «Nicht können». Die Ursachen ihrer Probleme fanden sie einerseits in ihrer Krankheit und/oder in der daraus resultierenden Therapie. Andererseits erwähnten sie auch organisatorische und institutionelle Hindernisse. Da die Patient(inn)en wenig Unterstützung erhielten, um aus diesem Spannungsfeld herauszukommen, nahmen sie die Suche nach Lösungen selbst in die Hand.

Auf der persönlichen Ebene zeigte sich, dass die Patient(inn)en bei der Bewältigung ihrer Ernährungsprobleme sehr pragmatische Lösungen anstrebten.

Sie experimentierten mit Nahrungsmitteln und ließen weg, was ihnen nicht schmeckte oder Schmerzen bereitete. Weil ihnen der Verstand sagte, dass sie essen müssen, versuchten sie Emesis und Nausea zu überwinden. Jeden Tag war neu zu entscheiden, was und wie viel sie trotz Schwierigkeiten essen wollten. Dazu benutzten sie ihre mentalen, körperlichen und wenn möglich ihre sozialen Ressourcen.

Lorig und Kollegen beschreiben die Fähigkeit des Selbst-Managements und halten in einer ihrer Studien fest, dass betroffene Menschen genügend Wissen erhalten müssen, um ihre Probleme zu identifizieren und zu lösen (Lorig, Halsted \& Holman, 2003). Sie beschreiben, dass Pflegepersonen verschiedene Rollen einnehmen müssen, um betroffene Menschen zu befähigen, mit ihren Herausforderungen zu leben. Weil diese Rollen durch die Pflegepersonen ungenügend eingenommen werden, führt dies zur Problematik der unbefriedigenden Beratung. Dass Pflegepersonen $\mathrm{zu}$ wenig pathophysiologisches Wissen über einschränkende Symptome bei einer onkologischen Therapie haben und deshalb nicht adäquat reagieren können, bestätigen Churm, Andrew, Holden, Hildreth und Hawkins (2009). Ein Mangel an Wahrnehmung und an Wissen bezüglich Malnutrition oder verminderter Nahrungsaufnahme während eines Spitalaufenthalts stellen auch Ross, Mudge, Young und Banks (2011) in ihrer Studie fest. Alle Pflegepersonen der Abteilungen, auf denen das Malnutritionsprogramm etabliert ist, erhalten zur Gewährleistung der Nachhaltigkeit regelmäßig Schulung zum Malnutritionsprogramm. Es ist jedoch bekannt, dass es nicht ausreicht, lediglich ein Instrument zu implementieren und Schulungen anzubieten, um die Nahrungsaufnahme bei Patienten zu steigern (Hickson, Conolly \& Whelan, 2011). Hickson und Kollegen sagen, dass die Verbesserung der Ernährungssituation durch Sensibilisierung und Leadership auf allen Ebenen der Spitalpolitik, zum Beispiel durch regelmäßige Evaluation und Qualitätsverbesserung der patientenbezogenen Ernährung, erfolgen muss.

Die Patient(inn)en stießen nicht nur auf der persönlichen Ebene an Grenzen, sie mussten auch betriebliche Schwierigkeiten überwinden. So versuchten sie ihre Nahrungsaufnahme zu optimieren, indem sie aus den angebotenen Menüs oder Menükomponenten auswählten. Sie versuchten aus den Angeboten etwas zusam- menzustellen, das ihnen helfen sollte, zu Kräften zu kommen. Dies gelang ihnen aber nur ungenügend. Ähnliche Beobachtungen machte Huber (2009) in ihrer Studie, in der sie festhält, dass es für Patient(inn)en mit Appetitproblemen schwierig sei, die Gerichte zu bestellen, welche sie essen konnten und die ihnen gut tun würden. Auch Mahadevan und Kollegen stellen in ihrer Studie fest, dass es für die Patient(inn)en sehr wichtig ist, eine Auswahl zu haben, um wieder Freude am Essen zu finden (Mahadevan, Hartwell, Feldman, Ruzsilla \& Raines, 2013).

Eine weitere Schwierigkeit waren die fixen Essenszeiten. So hätten sie lieber mehrere kleinere Mahlzeiten als drei große, da sie sich so schnell satt fühlten. Dass fixe Essenszeiten ein großes Problem darstellen zeigen zwei Studien auf (Iff et al., 2008; Naithani, Whelan, Thomas, Gulliford \& Morgan, 2008). Wie Iff et al. (2008) betonen, muss das Catering in der Lage sein, auf die individuellen Bedürfnisse der Patienten einzugehen. Naithani et al. (2008) zeigen in ihrer Studie auf, dass die Patient(innen) hungrig waren, weil die Essensabstände zu groß und die Bestellzeiten begrenzt waren. Auch entsprachen die Essenszeiten nicht ihren Gewohnheiten. Interessanterweise haben in unserer Studie nur zwei Patient(inn)en den großen Abstand zwischen Abendessen und Frühstück bemängelt. Die anderen Teilnehmer(inn)en waren von Hause aus eine lange Essenspause gewohnt.

Auffallend war der Umgang mit den Trinknahrungen, die als Zwischenmahlzeiten angeboten wurden. Nur zwei Patient(inn)en schmeckten diese; den anderen waren sie zu faserig, zu süß, zu dickflüssig. Alle wussten, dass Trinknahrung sinnvoll ist, aber sie hatten Mühe mit der Einnahme. Danach fühlten sie sich gesättigt, sodass sie bei den regulären Mahlzeiten weniger Appetit hatten. Die gleichen Erkenntnisse gewannen auch Larsen und Uhrenfeldt (2012) aus ihrer Analyse von 13 qualitativen Studien. Die meisten der befragten Patient(inn)en in unserer Studie bevorzugten nahrhafte Suppen, da diese gut zu schlucken waren und sie ihnen das Gefühl gaben, etwas Richtiges gegessen zu haben. Alle Patient(inn) en in unserer Studie erhielten jedoch ausschließlich süße Trinknahrung. Vivanti und ihre Forschungsgruppe schreiben in ihrem Studienbericht, dass Essensvorlieben vom Gesundheitszustand, vom Geschlecht, von erlernten Essensmustern und den Vorstellungen und Überzeugungen über die Nahrungsmittel geprägt sind (Vivanti et al., 2008). Die Autor(inn)en schlussfolgern, dass ein patientenorientierter Mahlzeitenservice süße und salzige Lebensmittel beinhalten sollte, die zudem einfach zu konsumieren sind, damit sich die Nahrungsaufnahme bei Risikopatienten verbessern kann (Vivanti et al., 2008). Diese Ansicht findet sich bei Iff et al. (2008) bestätigt. Laut den Autor(inn)en gilt es, Ernährungsprobleme bei Patient(inn)en zu erkennen und mit geeigneten Maßnahmen individuell zu reagieren. Eine Expertengruppe des Europarates hat schon 2006 eine Stellungnahme und Empfehlungen der Eidgenössischen Ernährungskom- 
mission zur Malnutrition im Spital herausgegeben (Keller, Meier, Lüthy, Rosé \& Sterchi, 2006). Darin ist beschrieben, dass interprofessionelle Zusammenarbeit zwischen ärztlichem Dienst, Pflege, Apotheke, Ernährungsberatung, Küche und Controlling/Einkauf notwendig ist, um das Risiko einer Malnutrition im Spital zu senken. Eine der Empfehlungen lautet, die Behandlung der Malnutrition den Bedürfnissen der Patient(inn)en anzupassen. Es ist also notwendig, Informationen über die Nahrungsaufnahme und das Essverhalten der Patient(inn)en zu sammeln, um daraus bedürfnisorientierte, individuell passende Menüs zu entwickeln. Zu den Empfehlungen zählt auch, dass das Personal über die verschiedenen Kostformen und deren Einfluss auf den Ernährungszustand der Patient(inn)en informiert sein sollte. Es stellt sich die Frage, welche Schlüsselpersonen die Verantwortung für das Ernährungsmanagement übernehmen. Denn die Resultate machen deutlich, dass die Patient(inn)en wenig bis keine Beratung hinsichtlich der Menübestellung erhielten.

\section{Schlussfolgerungen}

Durch die Studie konnte das bis dato anekdotische Wissen zu Erfahrungen von Patient(inn)en mit den Intervention im Malnutritionsprogramm erstmals wissenschaftlich untermauert und durch die systematische Analyse der Patient(inn)enperspektive gefestigt werden.

Die Ergebnisse zeigen, dass sich Risikopatienten durch den NRS-2002 identifizieren lassen und sie dem Malnutritionsmanagement zugeführt werden können. Das Programm ist jedoch für Pflegepersonen unterschiedlicher Stufen mit Verantwortung verbunden, welche sie ungenügend wahrnehmen. Es wäre notwendig, die Pflegenden kontinuierlich in der Umsetzung des Wissens zu begleiten, damit sie ihre Fähigkeiten im Umgang mit den Ernährungsschwierigkeiten der Patient(inn)en weiterentwickeln und nutzen können. Sie müssten eine partizipative Zusammenarbeit mit den Patient(inn)en suchen, um gemeinsam einen geeigneten Menüplan zu entwerfen.

Die Resultate machen deutlich, dass das Erfragen der Essenswünsche eine Aufgabe der Pflegeassistent(inn)en oder der Fachfrauen/Fachmänner Gesundheit ist. Diese könnten auch als Thementräger(inn)en zum Einsatz kommen und dementsprechend geschult und begleitet werden, denn sie haben sehr wenig Wissen über die $\mathrm{Zu}$ sammenhänge zwischen Krankheiten und Ernährung. Das würde die diplomierten Pflegefachpersonen entlasten und die Berufsbilder der Pflegeassistenz sowie der Fachfrauen/Fachmänner Gesundheit aufwerten. Die Verantwortung für die Ernährung der Patient(inn)en würde den diplomierten Pflegefachpersonen obliegen und somit auch die Klärung von Fragen an den Schnittstellen.

Ein weiterer kritischer Punkt sind die organisatorischen Hindernisse. Risikopatienten sollten eine verbesserte in- dividuelle Nahrungszusammensetzung erhalten. Es müssten unter anderem nahrhafte süße und salzige Snacks oder Suppen als Zwischenmahlzeiten zur Verfügung stehen, welche die Patient(inn)en auch außerhalb der regulären Mahlzeiten bestellen können. Ein weiterer wichtiger Punkt besteht darin, dass Patient(inn)en ihre Essenzeiten und die Anzahl der Mahlzeiten individuell wählen können. Um den Ernährungszustand der Risikopatient(inn)en $\mathrm{zu}$ verbessern oder zumindest zu erhalten, wäre jedoch auch auf den Abteilungen eine größere Flexibilität bezüglich des Caterings erforderlich.

Um zu evaluieren, ob die Erfahrungen anderer Risikopatient(inn)en mit den Erfahrungen unserer Studienteilnehmenden vergleichbar sind, müsste eine standardisierte Befragung erfolgen.

Die Studie zeigt einige Schwachpunkte im Malnutritionsprogramm in einem Zentrumsspital auf. Das sind unter anderem die interdisziplinären und multiprofessionellen Schnittstellen, welche bezüglich der Kompetenzen ungenügend definiert sind. Gezielte, idealerweise interprofessionelle Schulungen sowie Befähigung der am Malnutritionsprogramm beteiligten Berufsgruppen durch eine Expertin/einen Experten würden helfen, die Wichtigkeit der Ernährung der Risikopatienten zu erkennen und berufsgruppenübergreifend, koordiniert und patientenorientiert angehen zu können.

\section{Limitation}

Obwohl alle Befragten an einer Erkrankung litten, welche zu einem Malnutritionsrisiko führte, war die Anzahl teilnehmender Patient(inn)en klein. Es ist deshalb möglich, dass keine vollständige Redundanz der Themen erreicht werden konnte. Allerdings gehörten die befragten Teilnehmenden unterschiedlichen Altersgruppen an, weshalb bei relativ wenigen Teilnehmenden eine breite Palette von Erfahrungen vorzufinden war. Jedoch konnten zu wenige Männer rekrutiert werden, als dass wir genderspezifische Aussagen bezüglich der Erfahrungen machen können. $\mathrm{Zu}$ Beginn der Studie gingen wir davon aus, wesentlich mehr Interviews durchführen zu können. Die Gründe für die geringe Zahl der Teilnehmenden waren zum Teil organisatorischer Art, beispielsweise andere Prioritäten auf den Abteilungen oder eine nicht immer systematische Durchführung des NRS-2002. Zudem waren etliche Risikopatienten präterminal. Diese wurden aus ethischen Gründen nicht befragt.

\section{Dank}

Wir bedanken uns bei Edith Trachsel und Dr. Caroline Kiss für ihre fachliche und persönliche Unterstützung und bei den Pflegeexpertinnen der Abteilungen für die Mithilfe bei der Rekrutierung der Patient(inn)en. 


\section{Beiträge der einzelnen Autorinnen}

Entwicklung Studienprotokoll, Datensammlung und -analyse, Manuskripterstellung: EHJ

Studienbegleitung und Review des Manuskripts: KF Studienbegleitung und Review des Manuskripts: IAF

\section{Literatur}

Beck, A. M.; Balknas, U. N.; Furst, P.; Hasunen, K.; Jones, L. et al. (2001). Food and nutritional care in hospitals: how to prevent undernutrition - report and guidelines from the Council of Europe. Clinical Nutrition, 20 (5), $455-460$.

Churm, D.; Andrew, I. A.; Holden, K.; Hildreth, A. J.; Hawkins, C. (2009). A questionnaire study of the approach to the aneroxiacachexia syndrom in patients with cancer by staff in a district general hospital. Journal Support Care Cancer, 17, 503- 507.

Correia, M. I. T. D; Waitzberg, D. L. (2003). The impact of malnutrition on morbidity, mortality, length of hospital stay and costs evaluated through a multivariate model analysis. Clincal Nutrition, 22 (3), $235-239$

Ferguson, M.; Capra, S.; Bauer, J.; Banks, M. (2001). Development of a patient satisfaction survey with inpatient clinical nutrition services. Australian Journal of Nutrition and Dietetics, 58 (3), $157-163$.

Frei, A. (2006). Mangelernährung im Spital - medizinische Kosten und Kosteneffektivität bei Verhinderung. Bern: Bundesamt für Gesundheit.

Hickson, M.; Conolly, A.; Whelan, K. (2011). Impact of protected mealtimes on ward mealtime environment, patients experience and nutrition intake in hospitalised patients. Journal of Human Nutritions and Dietetics, 24, 370-374.

Huber, E. (2009). Essen im Spital - eine interdisziplinäre Herausforderung. Pflege, 22, $361-370$.

Iff, S.; Leuenberger, M.; Rösch, S.; Knecht, G.; Tanner, B. Stanga, Z. (2008). Meeting the nutritional requirements of hospitalized patients: An interdisciplinary approach to hospital catering. Clinical Nutrition, 27, $800-805$.

Imoberdorf, R.; Meier, R.; Krebs, P.; Hangartner, P. J.; Hess, B. et al. (2010). Prevalence of undernutrition on admission to Swiss hospitals. Clinical Nutrition, 29 (1), $38-41$.

Johansen, N.; Kondrup, J.; Plum, L. M.; Bak, L.; Norregaard, P. et al. (2004). Effect of nutritional support on clinical outcome in patients at nutritional risk. Clinical Nutrition, 23 (4), 539 - 550.

Keller, U.; Meier, R.; Lüthy, J.; Rosé, B.; Sterchi, A-B. (2006). Mangelernährung im Spital - Stellungnahme einer Expertengruppe des Europarates und Empfehlungen der Eidgenössischen Ernährungskommission. Schweizerische Ärztezeitung, 87 (19), $826-831$.

Kondrup, J.; Johansen, N.; Plum; L. M.; Bak, L.; Larsen, I. H. et al. (2002). Incidence of nutritional risk and causes of inadequate nutritional care in hospitals. Clinical Nutrition, 21 (6), 461 - 468.
Kondrup, J.; Allison, S. P.; Elia, M.; Vellas, B.; Plauth, M. (2003a). ESPEN guidelines for nutrition screening 2002. Clinical Nutrition, 22 (4), $415-421$.

Kondrup, J.; Rasmussen, H. H.; Hamberg, O.; Stanga, Z. (2003b). Nutritional risk screening (NRS 2002): a new method based on an analysis of controlled clinical trials. Clinical Nutrition, 22 (3), $321-336$.

Kyle, U. G.; Pirlich, M.; Schuetz, T.; Luebke, H. J.; Lochs, H.; Pichard, C. (2003). Prevalence of malnutrition in 1760 patients at hospital admission: a controlled population study of body composition. Clinical Nutrition, 22 (5), $473-481$.

Kyle, U. G.; Pirlich, M.; Schuetz, T.; Lochs, H.; Pichard, C. (2004). Is nutritional depletation by Nutritonal Risk Index associated with increased length of hospitals stay? A population-based study. Journal of Parenteral and Enteral Nutrition, 28 (2), $99-104$.

Larsen, L. K.; Uhrenfeldt, L. (2012). Patient's lived experiences of a reduced intake of food and drinks during illness: a literature review. Scandinavian Journal of Caring Science, 27, 184-194.

Lorig, K. R.; Halsted, R.; Holman, M. D. (2003). Self-Management Education: History, Definition, Outcomes and Mechanism. Annuals of Behavioral Medicine, 26 (1), 1 - 7.

Mahadevan, M.; Hartwell, H. J.; Feldman, C. H.; Ruzsilla, J.; Raines, E. R. (2013). Assisted-living elderly and mealtime experience. Journal of Human Nutrition and Dietetics, 27, 152-161.

Mayring, Ph. (2010). Qualitative Inhaltsanalyse: Grundlagen und Techniken. Landsberg: Beltz.

Mclntyre, A. (2008). Participatory Action Research (Vol. 52). California:Thousands Oaks: Sage Publications.

Naithani, S.; Whelan, K.; Thomas, J.; Gulliford, M. C.; Morgan, M. (2008). Hospital inpatients' experiences of access to food: a qualitative interview and observational study. Journal Health Expectations, 11 (3), $294-303$.

Ockenga, J.; Freudenreich, M.; Zakonsky, R.; Norman, K.; Pirlich, M.; Lochs, H. (2005). Nutritional assessment and management in hospitalised patients: implication for DRG-based reimbursement and health care quality. Clinical Nutrition, 24 (6), 913-919.

Ross, J. L.; Mudge, A. M.; Young, A. M.; Banks, M. (2011). Everyone's problem but nobody's job: Staff perceptions and explanations for poor nutritional intake in older medical patients. Journal of Nutrition and Dietetics, 68,41-46.

Sorensen, J.; Kondrup, J.; Prokopowicz, J.; Schiesser, M.; Kraehenbuehl, L. et al. (2008). EuroOOPS: An international, multicentre study to implement nutritional risk screening and evaluate clinical outcome. Clinical Nutrition, 27, 340-349.

Stratton, R. J.; Elia, M. (2007). Who benefits from nutritional support: what is the evidence? European Journal of Gastroenterology and Hepatology, 19 (5), 353 - 358.

Van Venrooij, L. M.; De Vos, R.; Borgmeijer-Hoelen, M. M.; Haaring, C.; De Mol, B. A. (2008). Preoperative unintended weight loss and low body mass index in relation to complications and length of stay after cardiac surgery. The American Journal of Clinical Nutrition, 87 (6), 1656 - 1661.

Vivanti, A.; Banks, M.; Aliakbari, J.; Suter, M.; Hannan-Jones, M.; McBride, E. (2008). Meal and food preferences of nutritionally at-risk inpatients admitted to two Australian tertiary teaching hospitals. Journal of Nutrition and Dietetics, 65, $36-45$. 


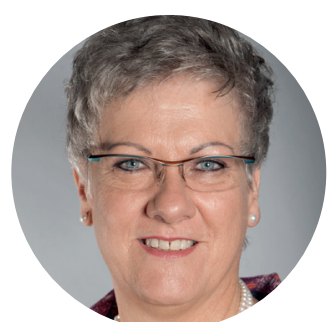

Elisabeth Haldemann-Jenni

\section{Dr. Irena Anna Frei}

Abteilung Praxisentwicklung Pflege

Ressort Pflege/MTT

Universitätsspital Basel

Hebelstrasse 2

4053 Basel

Schweiz

irenaanna.frei@usb.ch

\section{Was war die größte Herausforderung bei Ihrer Studie?}

Die grösste Herausforderung war der knappe Zeitplan für die Durchführung und Analyse der Interviews. Ich hatte einen grossen Respekt vor der Erlebniswelt der Teilnehmenden, so dass mein grösstes Anliegen war, diese Erlebniswelt korrekt darzustellen.

Was wünschen Sie sich bezüglich der Thematik für die Zukunft?

Ich wünsche mir, dass das Thema Malnutrition in den Fokus des medizinischen Personals kommt und dadurch malnutrierte oder RisikoPatient(inn)en gezielter und individueller in ihrer Nahrungsaufnahme unterstützt werden, so dass sie ihren Ernährungszustand erhalten oder verbessern können.

Was empfehlen Sie den LeserInnen zum Weiterlesen/Vertiefen? Ich empfehle den Leser(inne)n, dass Sie sich mit der Ernährungsliteratur auseinander setzen, dafür gibt es zahlreiche gute Bücher oder Artikel. Ich empfehle den Leser(inne)n, dass sie die

Ernährungsberater(innen) nach geeigneten Studien, Artikel und Bücher fragen. Die Ernährungsberater(innen) können sie auf den neusten Stand der evidenzbasierten Ernährung bringen.

Manuskripteingang: 26.08.2015

Manuskript angenommen: 20.10.2015 